\title{
A new generation of hollow polymeric microfibers produced by gas dissolution foaming
}

Received 00th January 20xx, Accepted 00th January 20xx DOI: $10.1039 / x 0 x \times 00000 x$

\author{
Suset Barroso-Solares ${ }^{a, b, c,}{ }^{,}$, Daniel Cuadra-Rodriguez ${ }^{a}$, Maria Luz Rodriguez-Mendez ${ }^{b, c}$, Miguel Angel \\ Rodriguez-Perez ${ }^{\mathrm{a}, \mathrm{b}}$, and Javier Pinto ${ }^{\mathrm{a}, \mathrm{b},{ }^{*}}$
}

\begin{abstract}
A new and straightforward route to produce polymeric hollow microfibers is proposed. Polycaprolactone (PCL) hollow fibers are obtained for the first time by an environmentally friendly gas dissolution foaming approach, overcoming its limitations to induce porosity on samples on the micrometric range. Different porous morphologies are achieved from solid PCL microfibers with a well-controlled diameter obtained by conventional electrospinning. The optimization of the foaming parameters provides two sets of well-defined hollow fibers, one showing smooth surfaces and the other presenting an enhanced surface porosity. Accordingly, gas dissolution foaming proves to be not only suitable for the production of hollow polymeric microfibers, but also capable to provide diverse porous morphologies from the same precursor, solid fibers. Moreover, a preliminary study about the suitability of this new generation of foamed hollow polymeric fibers for drug delivery is carried out, aiming to take advantage of the enhanced surface area and tunable morphology providing by the proposed new production method. It is found that the foamed microfibers can be loaded up to 15 wt.\% of Ibuprofen while preserving the morphology of each kind of fibers. Then, foamed PCL fibers presenting a hollow structure and surface porosity shows a remarkable constant Ibuprofen release for almost one day and a half. In contrast, the original solid fibers do not present such behavior, releasing all the Ibuprofen in about seven hours.
\end{abstract}

\section{1-Introduction}

Polymeric fibers produced by electrospinning have attracted significant attention in the last years due to their exceptional features in terms of their diameter in the micrometric or nanometric range and their high surface areas. ${ }^{1,2}$ The versatility of the production technique and the wide availability of polymers suitable allowed successfully employing electrospun polymeric fibers on diverse applications such as tissue engineering scaffolds and site-specific drug delivery, ${ }^{1-9}$ water treatment, ${ }^{1,10-12}$ sound absorption, 13 sensors, ${ }^{2}$ and catalysis. ${ }^{2}$

The large surface area, regarding their volume, of these materials is mainly related to the diameter of the fibers and the presence of porosity, which can be limited to the surface of the fibers or their hole volume. On the one hand, different techniques have been proposed to enhance the surface porosity, and therefore the surface area, of electrospun polymer fibers, such as the selective removal of a component of composite or blend fibers, or the use of a phase separation approach of different polymers during the electrospinning process. ${ }^{2}$ Both methods require the use of specific spinning parameters, not comparable to those of solid fibers Another approach is to induce the presence of surface porosity by

a. Address here.

+ Footnotes relating to the title and/or authors should appear here.

Electronic Supplementary Information (ESI) available: [details of any supplementary information available should be included here]. See DOI: 10.1039/x0xx00000x controlling the solvent evaporation or ambient humidity, but without achieving an accurate control on the formation of this porosity. ${ }^{2}$ On the other hand, hollow polymeric fibers have been obtained by complex procedures of co-electrospinning by using immiscible blends and then removing selectively one of the phases, $2,6,14,15$ or by extrusion. ${ }^{16-18}$ In both cases, the porosity is achieved by the removal of one phase once the fiber has been formed, and require the tuning of the electrospinning parameters or setup. Co-extrusion processes have also proved to be an efficient route to produce hollow fiber polymeric membranes for gas separation, providing diverse porosities or even enhanced surface porosity by modifying the fabrication parameters. ${ }^{16,19,20}$ Therefore, for the same initial polymer, different electrospinning setups or processing parameters are required to provide diverse porosities.

On the contrary, foaming techniques are commonly employed to induce the formation of well-controlled and tunable porosity inside polymer samples. ${ }^{21}$ In particular, the gas dissolution foaming technique is well-known as a cost-efficient and environmentally friendly technique to produce microporous and nanoporous polymer foams from a wide range of polymers, both amorphous and semicrystalline, in which the final features of the porosity can be controlled by the foaming parameters. ${ }^{22-24}$ In this procedure, pressurized $\mathrm{CO}_{2}$ or $\mathrm{N}_{2}$ is dissolved into a polymer matrix at a controlled pressure and temperature with the aim to induce the formation of pores after the pressure release. The thermodynamic instability induced by the pressure drop promotes the formation of small nuclei, which can grow into pores if the polymer matrix is heated up to temperatures near or over the glass transition or melting temperature of the polymer..$^{22-24}$ 
However, this technique could not be employed on samples with dimensions on the micrometric range, such as electrospun polymeric fibers, as once the pressure is released, the gas can quickly diffuse out of micrometric samples without inducing the formation of nuclei. ${ }^{25}$ Nevertheless, the incorporation of diffusion barriers around polymeric micro and nano-particles has recently allowed inducing the formation of porosity on that materials by gas dissolution foaming. These barriers increased the time required by the gas to diffuse out the sample, allowing the nucleation and pore growth to happen. ${ }^{25}$ Therefore, the use of gas diffusion barriers could overcome the limitations of the gas dissolution foaming when applied on polymeric microfibers, allowing a fine an independent control over their main features, such as diameter and porosity, never achieved before.

Among other applications, the tailoring of the porosity and structure of polymeric microfibers has been proposed to improve the drug delivery capability of such materials. ${ }^{26-28}$

Both the dosage and delivery procedure of a therapeutic agent into our organism are known to be key parameters to provide highefficient and safe medical treatments. ${ }^{29,30}$ However, most of the widely used conventional delivery systems (e.g., pills, tablets, drops, injections, etc.) do not fulfill these requirements, as they usually present a poorly controlled first-order release (i.e., a decreasing release rate with time). The main drawbacks associated with this kind of release are the potential toxicity if the dosage released at the beginning of the treatment is too high, and low efficiency if the total dosage administrated should be kept low to prevent that potential initial toxicity. ${ }^{29,30}$ In particular, the optimization of polymer-based drug delivery devices has provided systems with promising constant drug release rates but generally limited to release times below 24 hours. ${ }^{29}$ Moreover, some of the polymers providing a controlled drug delivery capability (e.g., polyethylene, polypropylene, polydimethylsiloxane) are not biodegradable, ${ }^{31}$ being necessary to develop new polymer-based systems able to overcome these drawbacks. 8,29,30,32

Among other treatments, wound-dressing could benefit from the development of improved polymer-based devices, such as porous electrospun polymeric fiber mats with drug delivery capability. ${ }^{7-9,33,34}$ The improvement of these devices, for instance, achieving longlasting controlled painkiller deliver rates could help millions of people who are burned severely every year, requiring medical attention, or suffer from chronic skin ulcers. ${ }^{9}$ Moreover, it has been proposed that the delivery of anti-inflammatory factor and painkillers locally by using fibrous dressings can improve the patient's comfort while avoiding risks or side effects related to their systemic delivery. ${ }^{35,36}$

Herein, a new environmentally-friendly approach to produce hollow polymeric microfibers with fine control over their final morphology and porosity is proposed. PCL was selected as the polymeric base of the electrospun fibers due to its biocompatibility, nontoxicity, biodegradability, and adequate mechanical properties. ${ }^{3,4,8,9,14,33,34,37}$ Then, PCL microfibers mats were obtained by conventional electrospinning and subsequently modified by a gas dissolution foaming process. ${ }^{24,38}$ The introduction of a removable gas diffusion barrier during the foaming procedure allows obtaining for the first time hollow polymeric microfibers with tunable surface porosity by a gas dissolution foaming approach. In addition, the adjustment of the foaming parameters provides hollow fibers with diverse morphologies from the same solid precursor fibers, a unique feature beyond the reach of other hollow fibers manufacturing techniques. Then, a common anti-inflammatory and painkiller factor such as Ibuprofen was successfully loaded into a variety of solid and hollow fibrous mats by supercritical $\mathrm{CO}_{2}$ impregnation. Finally, the drug delivery capability of the different fibers was characterized, finding that the tuning of the porous structure of the fibers provides a controlled rather constant delivery rate of the Ibuprofen for almost 40 hours. Therefore, the proposed new production procedure proved the be a versatile approach with the capability to vary the morphology of the hollow fibers, to enhance their performance according to the selected application.

\section{2-Results and discussion}

\section{1-Electrospun PCL fibers.}

First, the morphology solid PCL fiber mats obtained by electrospinning were studied by SEM (Fig. 1). Fiber mats with a quite homogeneous thickness distribution, showing an average value of $6.5 \pm 0.7 \mu \mathrm{m}$, were obtained (Fig. 1.a). Also, close-up cross-section micrographs of the obtained fiber mats shown that the individual fibers are solid, and present a quite smooth surface with scarce surface porosity, produced by the solvent evaporation during the fibers formation in the electrospinning process (Fig. 1.b). ${ }^{2}$ Moreover, the solid PCL fiber mats presented a BET surface area of $1.696 \mathrm{~m}^{2} / \mathrm{g}$.

\section{2-Gas dissolution foaming of electrospun PCL fiber mats}

Samples of both pristine and PVOH-imbibed electrospun PCL fibers (see Supporting Information S.I-1) were subjected to the gas dissolution foaming process using pressurized $\mathrm{CO}_{2}$ at $30 \mathrm{MPa}$ and RT or $45^{\circ} \mathrm{C}$ for 24 hours. As expected, pristine PCL fibers did no present porosity after any of these procedures, as the $\mathrm{CO}_{2}$ diffuses out of the micrometric fibers faster than the mechanisms inducing the formation of pores (i.e., nucleation and growth). ${ }^{25,38}$ On the contrary, PVOH-imbibed fiber mats successfully foam at both saturation temperatures, obtaining the first generation of gas dissolution foamed polymeric microfibers reported in the literature. Prior to SEM observation of the foamed fiber mats, the surrounding $\mathrm{PVOH}$ was removed immersing the imbibed fibers in an ultrasonic hot water bath. Once the fibers mats seem free of $\mathrm{PVOH}$, the surface of individual fibers was studied by microRaman Spectrometry and compared to those of pristine PCL fibers.
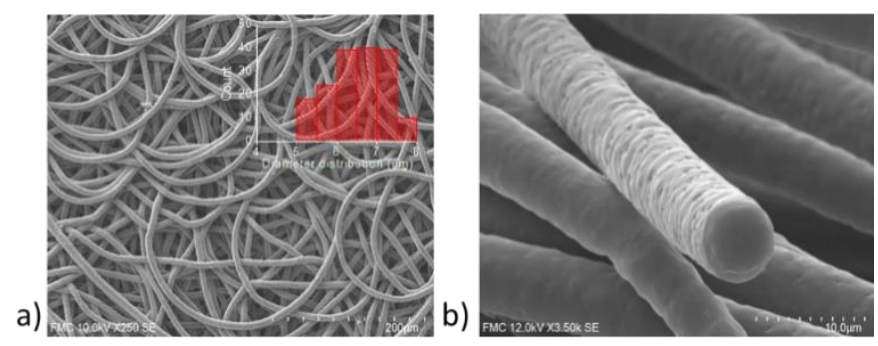

Fig. 1. SEM micrographs of the solid PCL fiber mats obtained by electrospinning. Thickness histogram of the fibers is shown on an inset (a). 
It was found that, although the complete absence of small residues of PVOH within the fiber mats cannot be assured, the surface of the individual foamed PCL fibers is successfully exposed after the PVOH removal (see Supporting Information S.I-2).

SEM observation of the foamed fiber mats confirmed the preservation of the fiber mats, as well as the generation of pores, but also clearly distinguish features between the fibers foamed at different temperatures (Fig. 2). On the one hand, fibers processed using a saturation temperature of $45^{\circ} \mathrm{C}$ spontaneously foam after being extracted of the pressure vessel, presenting a hollow structure and increased surface porosity regarding the solid precursor PCL fibers (Fig. 2.a and 2.b, from now on these fibers will be referred as hollow+porous fibers). The expansion induced by the foaming procedure is noticed in the thickness distribution of the fibers (see inset of Fig. 2.a), showing an average thickness of $11.2 \pm 1.9 \mu \mathrm{m}$. Another characteristic feature of these hollow+porous fibers is that the external walls of the fibers are quite thin, of about hundreds of nanometers (Fig. 2.b), and present well-defined pores with an average size about $740 \mathrm{~nm}$. On the other hand, fibers processed using RT as saturation temperature required to be immersed in a heated bath at $60^{\circ} \mathrm{C}$ for 1 minute to complete the foaming process. The obtained foamed fibers also present a hollow structure, but no increased surface porosity (see Fig. 2.c and 2.d, from now on these fibers will be referred as hollow fibers). As shown by the thickness distribution of the hollow fibers (see inset of Fig. 2.c), these fibers also expanded, presenting an average thickness of $11.0 \pm 1.3 \mu \mathrm{m}$. In this case, the walls of the hollow fibers are thicker, with thicknesses over the micron (Fig. 2.d).

Regarding the processing temperatures and final structures, it is possible to briefly discuss the mechanisms involved in the development of the fibers' porosity. Hollow+porous fibers spontaneously foam after the pressure release, as the saturation temperature $\left(45^{\circ} \mathrm{C}\right)$ should be higher than the effective melting point of $\mathrm{PCL}$, decreased due to the plasticization effect of $\mathrm{CO}_{2}{ }^{39}$ tending to coalescence into the hollow structure due to hydrostatic forces. ${ }^{40}$
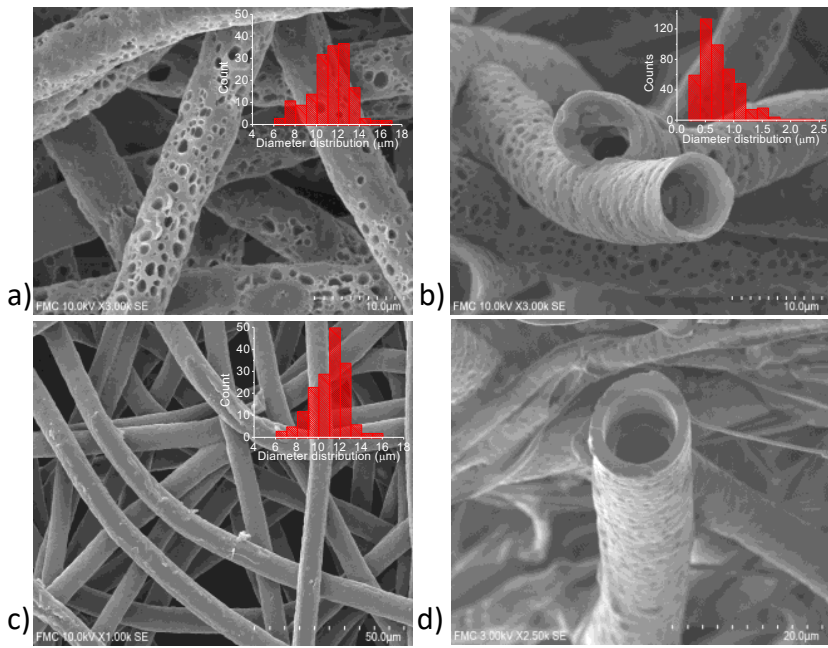

Fig. 2. SEM micrographs of the hollow+porous $(\mathbf{a}, \mathbf{b})$ and hollow (c, d) PCL fiber mats obtained by gas dissolution foaming. Thickness histogram of the foamed fibers $(\mathbf{a}, \mathbf{c})$ and surface pore size histogram of the hollow+porous fibers (b) are shown on insets.
Optimization of the processing parameters allows narrowing the effective melting point to about $45^{\circ} \mathrm{C}$, as no spontaneous foaming is found below that saturation temperature, see Supporting Information S.I-3.

Then, the quick cooling of the samples during the pressure release and the $\mathrm{CO}_{2}$ diffusion out of the samples, slow due to the PVOH barrier but not negligible, allows the stabilization of the hollow+porous structure of the fibers. This spontaneous foaming allows the formation and growth of pores inside the fibers and in their surfaces (probably promoted by the presence of an interface), ${ }^{41}$ with the internal pores. On the contrary, hollow fibers require an additional heating after the pressure release to induce the formation of the pores (i.e., the effective melting point of the $\mathrm{PCL}$ is over the saturation temperature). This heating step was performed at $60^{\circ} \mathrm{C}$ for 1 minute, which means that the plasticized PCL is expected to become melted entirely during the process. Moreover, during the time required to extract the samples from the pressure vessel and to introduce them into the heated bath, there will be some $\mathrm{CO}_{2}$ diffusion out of the external parts of the fibers. ${ }^{25}$ Both effects hinder the formation of pores in the outer surfaces of the fibers and promote the coalescence of the inner pores into a well-defined hollow structure. ${ }^{40}$

SEM micrographs of the foamed fibers (Fig. 2) clearly showed an increased surface area concerning the initial solid PCL fibers (Fig. 1). The increased surface area provided by the foaming process was further confirmed by BET measurements. Hollow fibers presented a BET surface area of $26.994 \mathrm{~m}^{2} / \mathrm{g}$, whereas hollow+porous fibers had a slightly higher value of $28.337 \mathrm{~m}^{2} / \mathrm{g}$. Therefore, the foaming process increased the BET surface area of the fibers more than 15 times (see section 2.1).

The obtained hollow PCL fibers, following for the first time a gas dissolution foaming approach, are comparable, or even better, than most of the previous works producing hollow polymeric fibers in terms of the obtention of hollow microfibers with well-controlled structure. Wang et al. ${ }^{14}$ obtained hollow PCL fibers by coelectrospinning with a similar range of diameters, between 8 to 15 $\mu \mathrm{m}$, but presenting a broader diameters distribution in all cases and not so well-defined morphologies. He et al. ${ }^{42}$ produced polyamideimide-based hollow fibers also by co-electrospinning with diameters of hundreds of microns and very thick outer walls. While De Rovere et al. ${ }^{43}$ presented polypropylene hollow fibers by melt-spun with diameter ranging between 40 and $60 \mu \mathrm{m}$. Also, the obtained fibers are superior in terms of the reduction of their diameter to hollow polymeric fibers obtained by co-extrusion or extrusion foaming processes, which typically present diameters of hundreds of microns or even over $1 \mathrm{~mm}$, while share with co-extrusion processes the capability to enhance the surface porosity of the fibers. ${ }^{16-20}$ Therefore, the obtained fibers could extend the range of application of current hollow fibers obtained by co-extrusion in fields such as gas separation or even be suitable for new applications.

\section{3-Ibuprofen impregnation tests}

Ibuprofen impregnation tests, assisted by $\mathrm{CO}_{2}$ pressurized at $15 \mathrm{MPa}$ and RT for 3 hours, were carried out using three kinds of PCL fibers, the pristine electrospun solid PCL fibers, and the foamed hollow+porous and hollow PCL fibers. The amount of Ibuprofen 
loaded into each kind of fibers was determined by thermogravimetric analysis (TGA, see Supporting Information S.I-4). The solid and hollow PCL fibers presented similar Ibuprofen contents of $11.5 \pm 2.8$ wt.\% and $12.6 \pm 2.0 \mathrm{wt} . \%$, respectively. While hollow+porous fibers showed a higher Ibuprofen load up to $15.2 \pm 1.2 \mathrm{wt} . \%$. These results indicate that perhaps the solid and hollow fibers were not completely saturated of Ibuprofen, due to the higher thicknesses of the PCL layers in which the Ibuprofen should be diffused than the holow+porous fibers (i.e., in the solid and hollow fibers Ibuprofen should diffuse through a PCL thickness respectively of about the radius of the fibers $(\sim 3.3 \mu \mathrm{m})$ or the external walls $(\geq 1 \mu \mathrm{m})$, while in the hollow+porous fibers the thickness is only about hundreds of nanometers). However, attempts to increase the impregnation time did not provide evident changes. In all the cases it was proved by SEM micrographs that the Ibuprofen impregnation procedure did not induce changes in the structure or morphology of the fibers (see Supporting Information S.I-4). Also, it was found that the pressurized air cleaning of the samples avoids the presence of precipitated Ibuprofen in the surface of the fibers (see Supporting Information S.I4).

The drug loading tests provided Ibuprofen loads higher than previously reported values of drug loading in hollow PCL fibers (9 wt.\% by blending), ${ }^{14}$ and in the same order of magnitude than those previously reported in the literature employing different polymer sheets such as polymethylmethacrylate (3-30 wt.\%) and polyvinylpyrrolidone (10-30 wt.\%), ${ }^{44,31}$ polylactic acid films (up to 40 wt.\%), ${ }^{45}$ or even PCL sheets (3-27 wt.\%). ${ }^{46}$ Particularly, the previous reports results using $\mathrm{PCL}$ sheets may suggest that further optimization of the Ibuprofen impregnation test could provide even higher Ibuprofen loads.

Moreover, the efficiency of the impregnation process was estimated by determining the partitioning coefficient $K$ (see Experimental section), obtaining a value of 27.2. As this coefficient usually ranges from $10^{-2}$ (inefficient drug loading, with the drug presenting much higher affinity by the $\mathrm{CO}_{2}$ than the polymer) to $10^{4}$ (high-efficient drug loading, with the drug presenting much higher affinity by the polymer than the $\mathrm{CO}_{2}$ ), it can be established that the performed Ibuprofen impregnation showed an intermediate efficiency. ${ }^{36}$ Also, an acceptable affinity between the Ibuprofen and the PCL matrix is evidenced by the diffusion of the lbuprofen into the PCL in a molecular form of forming small crystals (see Supporting Information S.I-5), which is expected to enhance its solubility in water without affecting their efficiency as painkiller. ${ }^{47}$

An additional advantage of performing this impregnation step using $\mathrm{CO}_{2}$ is that any potential toxic residue of organic solvents from the electrospinning process will be easily removed, avoiding potential risks associated to the use of electrospun fibers. ${ }^{8}$

\section{4-Ibuprofen delivery tests.}

A preliminary study of the drug delivery capability of the produced fibers was carried out by determining the cumulative Ibuprofen release of the solid, hollow, and hollow+porous PCL fibers in contact with water for 40 hours using UV-Vis spectroscopy. First, it was measured the total amount of Ibuprofen released for each kind of fibers and related to the exact mass of fibers employed on each test. It was found that the total amount of Ibuprofen released by each kind of fibers corresponds to the initial Ibuprofen load (i.e., about 11-12 wt.\% for solid and hollow fibers and about 15 wt.\% for hollow+porous fibers). Therefore, a complete Ibuprofen release from the fibers is achieved. This result was further confirmed by analyzing with TGA the fiber mats after the Ibuprofen release, finding no presence of Ibuprofen remains.

Accordingly, in order to compare the release kinetics of each kind of fibers, it was calculated the cumulative percentage of Ibuprofen released with time (Fig. 3).

Significant differences were found related to the structure of the fibers. Solid fibers presented the fastest release, reaching a complete Ibuprofen release in just $\mathbf{4 2 0}$ minutes, while hollow fibers required 1440 minutes to release their entire Ibuprofen load, and hollow+porous fibers kept the lbuprofen release until 2280 minutes. This behavior can appear to be counterintuitive, as faster release rates could be expected from higher surface areas. Therefore, further analysis is required to understand the underlying mechanisms.

First, the release profiles of the different fibers were analyzed using the Korsmeyer-Peppas model (Table 1)..$^{28,48}$ This model provided a proper fitting for the Ibuprofen release from both solid and hollow+porous fibers (e.g., $R^{2}$ values about 0.96 ), while hollow fibers are not so well described $\left(R^{2}=0.85\right)$. Regarding the information provided by this model about the potential release mechanisms, solid and hollow fibers present $n$ values related to non-Fickian diffusion. On the contrary, the Ibuprofen delivery of the hollow+porous fibers can be assimilated to a Fickian diffusion ( $n$ 0.45). However, as the PCL of the polymer matrices of all these fibers is not expected to suffer changes, neither are the diffusion mechanisms of the drug through the polymer.

Moreover, the rather constant release rate of the hollow+porous fibers cannot be associated with a degradation process of the biodegradable $\mathrm{PCL}$ fibers. It is known that the degradation of the polymer can lead to a rather constant release of the carried drug, but this process usually takes several days or even weeks, reaching a $100 \%$ drug release when the fibers are almost completely degraded, ${ }^{8,34}$ which is not the case in this work, as the fibers mats kept their integrity after the release tests. SEM micrographs of the fibers collected after the Ibuprofen release tests show some alterations on the external surface of the fibers, but the fibers are never close to complete degradation (Fig. 4).

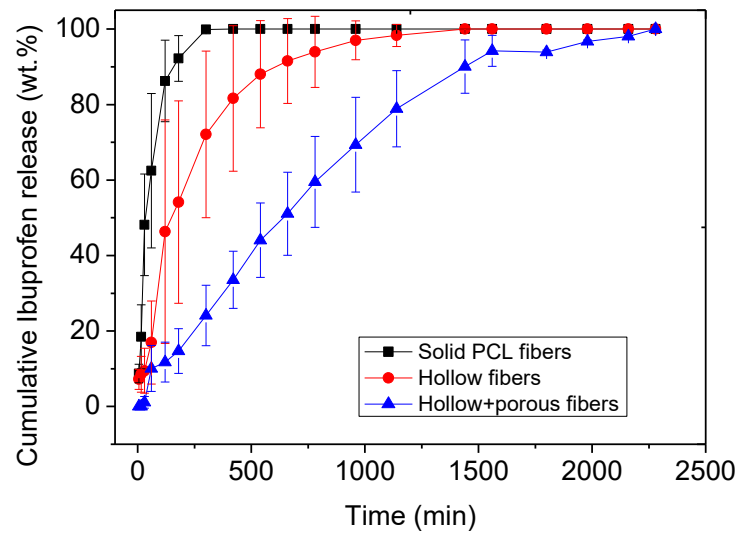

Fig. 3. Cumulative Ibuprofen release for 40 hours achieved with solid PCL fibers, hollow fibers, and hollow+porous fibers. 
Both solid and hollow PCL fibers presented a higher surface porosity due to the degradation of the outer layer of PCL (Fig.s 4.a and 4.b). At the same time, the hollow+porous fibers also present some alterations, but they seem to have suffer less degradation (Fig. 4.c), which could suggest a lower degree of interaction with the water during the tests.

Also, it is known that the morphology of the porous structure of a soluble scaffold or hollow fibers modifies their drug delivery capacity, by modifying the delivery mechanisms ${ }^{26-28}$, but in this case, the delivery mechanism does not seem to be altered. As the fiber mats kept their integrity, and the Ibuprofen is loaded into the PCL and not stored into the hollow structure or pores, the only delivery mechanism involved should be the diffusion of the lbuprofen from the PCL fibers to the surrounding environment.

Then, in order provide new insights about this behavior, the samples recovered after the Ibuprofen release tests were subjected again to the Ibuprofen impregnation procedure, as well as to the Ibuprofen release tests. No significant changes were found in terms of the Ibuprofen load of the different fibers, but the Ibuprofen delivery behavior presented some differences (Fig. 5).

Both solid and hollow PCL fibers presented a slower and poorly controlled release rate, while the hollow+porous PCL fibers present almost the same constant delivery rate than in the first Ibuprofen load and delivery cycle (i.e., the hollow+porous fibers could be even reusable, although reusability is not usually required for wounddressing or drug-delivery scaffolds).

Therefore, the features of the outer surface of the fibers seem to be related to both the lower degradation when exposed to water and the Ibuprofen delivery behavior.

It is well-know that a roughness increase of a surface modifies its wetting properties. ${ }^{49}$ In particular, for hydrophobic materials, such as $\mathrm{PCL}$, an increasing surface roughness can raise its hydrophobicity. This phenomenon is explained for the Cassie Model, in which the roughness promotes the presence of air pockets between the surface and the water drop, increasing the hydrophobicity of the surface and decreasing the contact between the water and the surface. ${ }^{50}$ As the hollow+porous fibers present a higher outer surface roughness due to its increased surface porosity, their lower Ibuprofen release rate and damage associated to the contact with water for 40 hours could be associated to a higher hydrophobicity of the surface of the individual fibers. This hypothesis also explains the changes in the second Ibuprofen load-release cycle, in which the fibers increasing their outer porosity/roughness due to the fiber degradation also decrease their Ibuprofen release rate.

Table 1. Application of the Korsmeyer-Peppas models to the Ibuprofen delivery of solid, hollow, and hollow+porous PCL fibers.

\begin{tabular}{llll}
\hline \multirow{2}{*}{ Samples } & \multicolumn{3}{l}{ Korsmeyer-Peppas Model } \\
& $\mathrm{K}$ & $\mathrm{n}$ & $\mathrm{R}^{2}$ \\
\hline Solid fibers & 0.19 & 0.84 & 0.966 \\
Hollow fibers & 0.18 & 0.61 & 0.855 \\
Hollow+porous fibers & 0.25 & 0.44 & 0.966 \\
\hline
\end{tabular}

a)
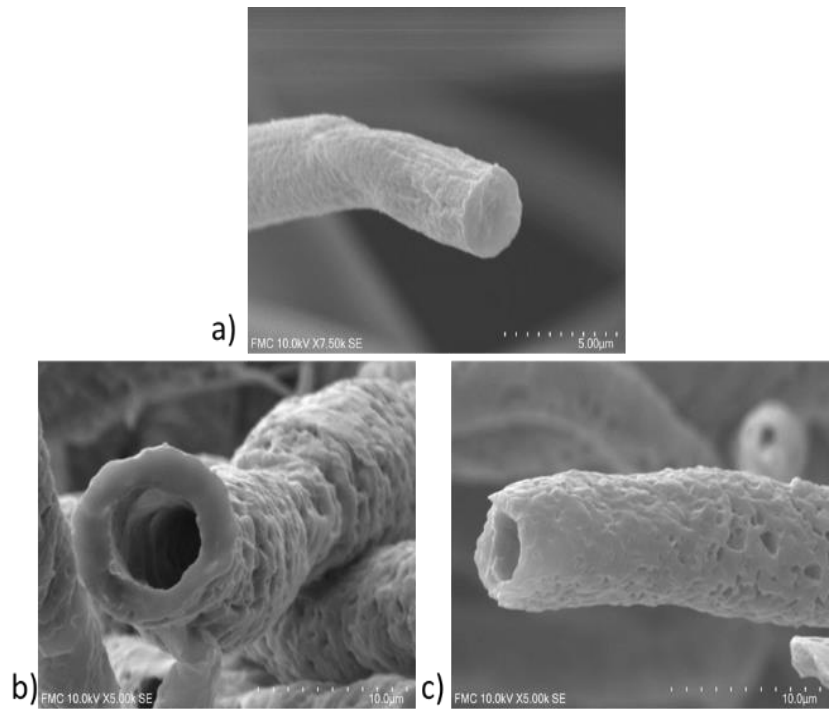

Fig. 4. SEM micrographs of the solid (a), hollow (b), and hollow+porous (c) PCL fibers after the Ibuprofen release tests.

With the aim to confirm this hypothesis the surface roughness of the solid, hollow, and hollow+porous PCL fibers was determined by Atomic Force Microscopy (AFM), finding a direct relationship between the increasing roughness of the fibers from the solid to the hollow+porous fibers with their decreasing average Ibuprofen release rates (Fig. 6 and Table 2).

As expected, the foaming process increases the ratio of the real rough surface area to the projected perfectly smooth surface $(r)$, showing the hollow+porous PCL fibers the highest ratio, in good agreement with the SEM and AFM micrographs of their surface (Fig. 2 and 6). Regarding the root mean square roughness $\left(R_{q}\right)$, both solid and hollow fibers present rough features of similar sizes on their surface ( 9-11 nm), while the hollow+porous fibers present an increased $R_{q}$ of about $16 \mathrm{~nm}$. Both roughness parameters are known to have a remarkable influence on the wetting properties of a surface, being directly related to the increase of the water contact angle of hydrophobic materials, such as PCL. ${ }^{51,52}$ Accordingly, the lower Ibuprofen release ratio of the hollow+porous fibers, and their higher resistance to degradation in contact with water, can be related to their enhanced surface roughness.

In view of the above results, the developed hollow+porous PCL fibers could be suitable candidates for advanced wound-dressings with a controlled drug-delivery rate for almost 40 hours, although further work should be performed to address their actual application. More importantly, the new production route proposed for hollow polymer fibers employs conventional industrial techniques, such as electrospinning and gas dissolution foaming, being possible to scaleup their production without requiring specific setups (e.g., coelectrospinning, co-extrusion). Also, this simple two-step route provides significant advantages in the tailoring of the hollow fibers, allowing a potential control of their diameter following conventional adjustments of the electrospinning process, while the features of the final porosity can be controlled independently controlled in the foaming stage. 


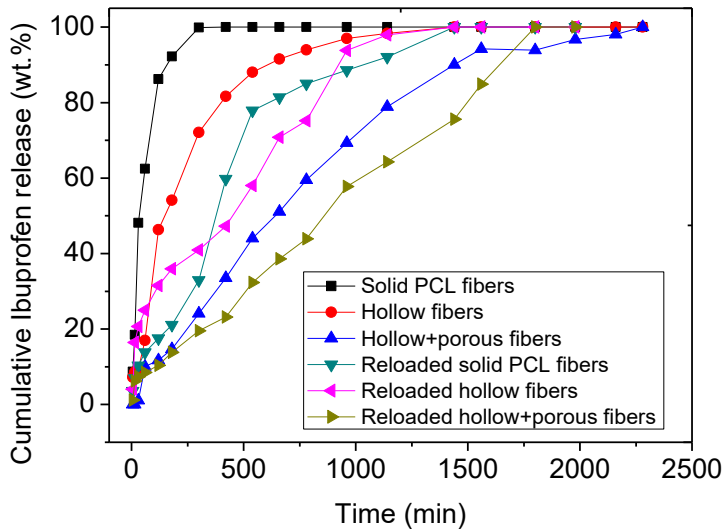

Fig. 5. Cumulative Ibuprofen release for 40 hours achieved with solid PCL fibers, hollow fibers, and hollow+porous fibers for two consecutive Ibuprofen load and release cycles. Error bars are not shown to facilitate the reading of the graph.

Fig. 6. Surface roughness profiles of the solid, hollow, and

a)

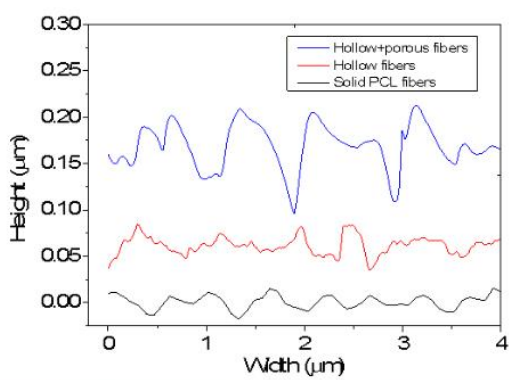

b)

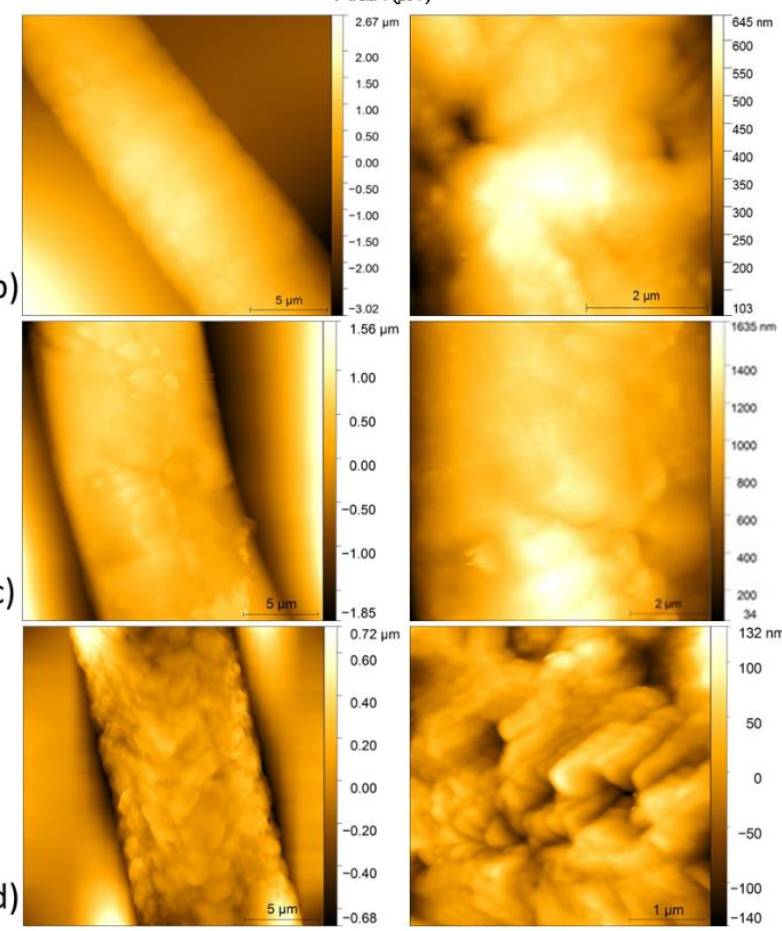

hollow+porous PCL fibers measured by AFM (a). AFM topographic micrographs of the surface of the solid (b), hollow (c), and hollow+porous PCL fibers(d).
Table 2. Surface roughness parameters and Ibuprofen average delivery rates of the solid, hollow, and hollow+porous PCL fibers.

\begin{tabular}{cccc}
\hline Sample & $r$ & $\mathrm{R}_{\mathrm{q}}(\mathrm{nm})$ & $\begin{array}{c}\text { Average } \\
\text { Ibuprofen } \\
\text { release rate } \\
(\mathrm{mg} / \mathrm{g} \cdot \mathrm{h})\end{array}$ \\
\hline Solid fibers & $1.009 \pm 0.004$ & $11.5 \pm 1.1$ & 16.4 \\
Hollow fibers & $1.013 \pm 0.004$ & $9.2 \pm 2.3$ & 5.3 \\
Hollow+porous fibers & $1.033 \pm 0.003$ & $16.3 \pm 3.0$ & 4.0 \\
\hline
\end{tabular}

Therefore, the proposed new route to produce hollow polymeric fibers is a promising approach for the development and optimization of these materials.

\section{3-Experimental}

\section{1-Materials}

Polycaprolactone (PCL) (Mn: $80000, \rho=1.15 \mathrm{~g} / \mathrm{cm}^{3}$, melting point $\left.\left(T_{m}\right)=61{ }^{\circ} \mathrm{C}\right)$ and high purity lbuprofen $(>98 \%)$ were purchased from Sigma-Aldrich (USA). Poly (vinyl alcohol) (PVOH) (Mowiflex C17, $\rho=$ $1.25 \mathrm{~g} / \mathrm{cm}^{3}$, glass transition temperature $\left(T_{\mathrm{g}}\right)=60^{\circ} \mathrm{C}, \mathrm{T}_{\mathrm{m}}=170^{\circ} \mathrm{C}$ ) was gently provided by Kuraray. Chloroform was purchased from Scharlab (Barcelona, Spain). Deionized water was employed in all the tests, and a medical-grade of carbon dioxide $\left(\mathrm{CO}_{2}\right)$ presenting $99.9 \%$ purity was employed in the foaming and gas impregnation processes.

\section{2-Fabrication of solid fibers}

PCL fibers were fabricated by a conventional electrospinning process. A PCL solution in Chloroform (10 wt.\%) was transferred into a syringe of $3 \mathrm{~mL}$ with a needle 21G. The syringe was placed in a syringe pump (NE-1000, New Era Pump Systems, Inc.) providing a flow rate of 1 $\mathrm{mL} \cdot \mathrm{h}^{-1}$. The voltage was fixed at $15 \mathrm{kV}$, and a copper target covered with aluminium foil was placed at a distance of $20 \mathrm{~cm}$ from the needle and used as a collector (a scheme of the process can be found in Figure S11, see Supporting Information). The thickness of the fiber mats was controlled by adjusting the deposition time, obtaining fiber mats with thicknesses of about $200 \mu \mathrm{m}$.

\section{3-Gas dissolution foaming}

Foaming experiments were performed according to the gas dissolution foaming process using carbon dioxide $\left(\mathrm{CO}_{2}\right)$ as a blowing agent ${ }^{53,54}$. In this procedure, the polymeric samples are introduced into a pressure vessel and subjected to pressurized $\mathrm{CO}_{2}$, at controlled conditions of pressure and temperature, until the complete gas saturation of the polymer. Then, the gas pressure is released, and the samples extracted from the pressure vessel. At this point, the samples could spontaneously expand due to the formation of pores if the saturation temperature is higher than the polymer melting point $\left(T_{m}\right)$ or glass transition temperature $\left(T_{g}\right)$. On the contrary, the samples are immersed into a heated bath to promote their temperature over their $\mathrm{T}_{\mathrm{m}}$ or $\mathrm{T}_{\mathrm{g}}$ and initiate the expansion of the 
pores (it should be noticed that the presence of $\mathrm{CO}_{2}$ on the polymer could decrease their effective $T_{m}$ or $T_{g}$ ). ${ }^{38}$ Following the approach proposed by Orsi et al. ${ }^{25}$, some of the fiber mat samples were coated with $\mathrm{PVOH}$ prior to the gas dissolution foaming procedure. This coating was performed by immersing the samples in a few $\mathrm{mL}$ of a $\mathrm{PVOH}$ solution in water ( $5 \mathrm{wt} . \%$ ) and then dry overnight at room temperature, obtaining a solid PVOH film (thickness about $300 \mu \mathrm{m}$ ) in which the fiber mats (with average thickness about $200 \mu \mathrm{m}$ ) were imbibed.

Then, both imbibed and pristine samples were saturated with $\mathrm{CO}_{2}$ within a high-pressure vessel of 1 liter (model PARR 4681) provided by Parr Instrument Company (Moline, IL, USA). The gas pressure is supplied and controlled by an accurate pressure pump controller (model SFT-10) provided by Supercritical Fluid Technologies Inc. (Newark, DE, USA). The vessel is equipped with a clamp heater of $1200 \mathrm{~W}$, and the temperature of the experiment can be recorded by a temperature controller (CAL 3300). Saturation pressure was kept during 24 hours at $30 \mathrm{MPa}$, while the saturation temperature varied from RT to $45^{\circ} \mathrm{C}$ (these parameters were previously optimized, see Supporting Information S.I-3). Then, the pressure was released by using an electro-valve, and the samples were extracted from the pressure vessel. Samples saturated at RT were immersed into a thermal bath (J. P. Selecta Model 6000685 ) at $60{ }^{\circ} \mathrm{C}$ for 1 minute to promote the foaming, while samples saturated at $45{ }^{\circ} \mathrm{C}$ foamed spontaneously after the pressure release. The bath liquid used was oil to prevent the dissolution of PVOH during foaming (a scheme of the process can be found in Figure S11, see Supporting Information).

After the foaming procedure, imbibed samples were immersed into an ultrasonic hot water bath for several cycles until the complete removal of the PVOH coating.

\section{4-Drug loading}

Again, $\mathrm{CO}_{2}$ was employed for the Ibuprofen impregnation of the fibers. In this $\mathrm{CO}_{2}$ impregnation procedure the $\mathrm{CO}_{2}$ diffuses into the polymer acting as a carrier of the drug, allowing the drug to locate inside the fibers. ${ }^{36,44,45}$ This procedure to load a polymeric sample with a drug present significant advantages such as that it can be carried out at low temperatures, due to the plasticization effect of the polymer induced by the $\mathrm{CO}_{2}$, preventing damage of thermo-labile drug molecules; and the absence of solvent residues. ${ }^{36,47}$ These tests were performed at $15 \mathrm{MPa}$ and RT for 3 hours. In this case, a small pressure vessel with a volume about $8 \mathrm{~cm}^{3}$ has been employed in order to minimize the amount of Ibuprofen required, and the pressurization and depressurization were carried out at a slow pace to avoid damage of the fibers (see Supporting Information S.I-4). Samples of each kind of fibers (e.g., solid, hollow, hollow+porous, when necessary after the removal of the PVOH) of about $1 \mathrm{mg}$ were introduced into the vessel together with an exceeding amount of Ibuprofen of about 0.2 grams (i.e., a much higher amount than the required to saturate both the pressurized $\mathrm{CO}_{2}$ and the fibers, which was about 0.05 grams for these experiments). After extracting the drug-loaded fibers from the vessel, the samples were cleaned with pressurized air to remove any residue of Ibuprofen which could have precipitated on the surface of the samples during the pressure release. ${ }^{36}$ Finally, the efficiency of the impregnation procedure can be evaluated by means of the partitioning coefficient $K$, which established the relative affinity of the drug for the $\mathrm{CO}_{2}$ and the polymer at the specific pressure and temperature of the test. This coefficient can be calculated using equation $1 .^{36}$

$$
K=\frac{C_{\text {polymer }}}{C_{\mathrm{CO}_{2}}}
$$

Where $C_{\mathrm{CO} 2}$ and $C_{\text {polymer }}$ are respectively the concentration at the equilibrium of the drug in the $\mathrm{CO}_{2}$ and the polymer phases, given in $\mathrm{g} / \mathrm{g}$. As the experimental Ibuprofen concentration in the $\mathrm{CO}_{2}$ could not be measured in our experiments, it was estimated from the data provided by Charoenchaitrakool et al. ${ }^{55}$.

\section{5-Characterization techniques}

The structure and morphology of the fibers were studied through Scanning Electron Microscopy (SEM) (HITACHI FlexSEM 1000) after being coated with gold, while the surface roughness of the fibers was analyzed by Atomic Force Microscopy in tapping mode using a Cypher ES AFM from Asylum Research. The data were acquired using a standard AC160TS cantilever from Olympus with a spring constant $k$ of approximately $26 \mathrm{~N} \mathrm{~m}^{-1}$, a $\mathrm{Q}$ factor of 300 and a resonant frequency of $260 \mathrm{kHz}$. The Gwyddion software was used for statistical analyses of surface characteristics and phase in AFM image. In particular, the ratio of the real rough surface area to the projected perfectly smooth surface $(r)$, and the root mean square roughness $\left(R_{q}\right)$ of the surface of the fibers were determined. Details about these parameters can be found elsewhere..$^{52}$ The roughness analysis was performed on $4 \mu \mathrm{m} \times 4 \mu \mathrm{m}$ topographic AFM micrographs, establishing a threshold of $1 \mu \mathrm{m}$ to discriminate the surface roughness from the waviness and shape of the surface of the fibers. The surface area of the solid and foamed fibers was measured by BET using a Surface Area and Porosity Analyzer ASAP 2420 (Micromeritics Instrument Corp.). The efficiency of the PVOH removal step after the foaming process was addressed by microRaman Spectrometry measurements on the surface of the fibers before being imbibed into the PVOH and after the PVHO removal, using a high-resolution Horiba-Jobin Yvon LABRAN HR 800 UV equipped with a Olympus BX41 microscope, a Symphony CCD detector, and $\lambda=532.8 \mathrm{~nm}$. The amount of Ibuprofen loaded into the fibers was determined by Thermo Gravimetric Analysis (TGA) (SDTA851, Mettler Toledo), heating the samples from 50 to $400{ }^{\circ} \mathrm{C}\left(5{ }^{\circ} \mathrm{C} / \mathrm{min}\right)$ in a nitrogen atmosphere. To ensure the complete degradation of the Ibuprofen, an isotherm was performed at $220^{\circ} \mathrm{C}$ for 2 hours, determining the Ibuprofen load as the weight loss percentage between about $100^{\circ} \mathrm{C}$ and the end of the isotherm (see Supporting Information S-I-4). Also, the Ibuprofen-loaded fibers were analyzed by Differential Scanning Calorimetry (DSC) performed using a Mettler-Toledo model DSC30 to determine if the loaded Ibuprofen is in crystalline or molecular form, following a heating ramp from -50 to $150{ }^{\circ} \mathrm{C}$ at $10^{\circ} \mathrm{C} / \mathrm{min}$ in a $\mathrm{N}_{2}$ atmosphere.

To study the Ibuprofen release behavior of the drug-loaded fibers, $1 \mathrm{mg}$ samples of each kind of fibers (e.g., solid, hollow, hollow+porous) were placed in a vial in $10 \mathrm{~mL}$ of distilled water and shaken at a constant rate of $200 \mathrm{rpm}$. After fixed periods of contact time (increasing from 5 to 120 minutes, until a total contact time of 2400 minutes) the samples were collected and placed in a new vial, while the remaining water was analyzed using an UV-visible scanning 
spectrophotometer (UV-2101PC, Shimazu). The concentration of Ibuprofen in the analyzed water was determined employing the 190 $\mathrm{nm}$ peak of the Ibuprofen, ${ }^{56}$ comparing the obtained results (e.g., $190 \mathrm{~nm}$ peak intensity) with a calibration line obtained from stock solutions of known concentration between 0.39 and $12.5 \mathrm{mg} / \mathrm{L}$. All the spectra were collected in the range $190-400 \mathrm{~nm}$, using distilled water as a reference sample. Once the concentration of Ibuprofen released by the fibers on each period was determined, it was possible to determine the total Ibuprofen load of the fibers, the release kinetics, the normalized cumulative release, and the average release rate as the ratio between the amount of Ibuprofen released $(\mathrm{mg})$ per gram of fibers and the time required to achieve a complete release. In addition, the Korsmeyer-Peppas model was employed to evaluate the Ibuprofen release. This model was developed to describe the drug release from a polymeric system, providing information about the drug release mechanism when employed to fit the first $60 \%$ of the drug release. This model can be expressed using equation $2 .{ }^{28,48}$

$$
\frac{M_{t}}{M_{\infty}}=K \cdot t^{n}
$$

Where $M_{t} / M_{\infty}$ is the fraction of drug release at time $t, K$ is the release rate constant, and $n$ is the release exponent. The value of this exponent might be employed to identify the release mechanisms for cylindrical shaped matrices (such as polymer fibers). In particular, values of $n=0.45$ correspond to Fickian diffusion, for $0.45 \leq n<0.89$ non-Fickian transport is expected, and for $n=0.89$ the drug delivery mechanism could be related to polymer swelling. As previously mentioned, the determination of $n$ should be carried out only for $M_{t} / M_{\infty}<0.6 .^{28,48}$

\section{Conclusions}

This work proposes a new approach, based on the gas dissolution foaming route, to produce PCL-based hollow microfibers. The use of an outer PVOH layer acting as a gas diffusion barrier, added after the production of solid PCL fibers by conventional electrospinning (solid fibers), allows the foaming of the microfibers by this technique. In particular, this barrier is able to keep the $\mathrm{CO}_{2}$ solved into the $\mathrm{PCL}$ enough time to allow the nucleation and growth of pores inside the microfibers. Moreover, the barrier is easily removed in hot water after the foaming procedure. Two different gas dissolution foaming procedures, usually known as one-step and two-step foaming, were successfully employed to obtain well-defined hollow fibers, also inducing significant differences on the morphology of the obtained hollow fibers. On the one hand, $\mathrm{PCL}$ fibers saturated with $\mathrm{CO}_{2}$ at 30 $\mathrm{MPa}$ and $45^{\circ} \mathrm{C}$ spontaneously foamed after being extracted from the pressure vessel, providing also a remarkable increase of the surface porosity of the fibers (hollow+porous fibers). On the other hand, PCL fibers saturated with $\mathrm{CO}_{2}$ at $30 \mathrm{MPa}$ and $\mathrm{RT}$ required of an additional foaming step, carried out for 1 minute in a heated bath at $60{ }^{\circ} \mathrm{C}$, to induce the foaming, showing a hollow structure with thicker walls and no increase of the surface porosity (hollow fibers). In addition to the presence of the porous structure, the foamed fibers also presented higher outer diameters, as a result of the expansion procedure induced by the foaming procedure, and an increased surface area.
As PCL is a biodegradable and biocompatible polymer, it is of great interest in biomedical applications such as in-situ local drug delivery. Then, the suitability of the obtained fibers for drug delivery applications was studied. First, it was proved that all the fibers, solid, hollow, and hollow+porous, can be loaded with a conventional painkiller and anti-inflammatory such as Ibuprofen. All the fibers achieved drug loads about or over 10 wt.\% after 4 hours of impregnation assisted by $\mathrm{CO}_{2}$, presenting the hollow+porous fibers the higher load (15 wt.\%). As the characteristic morphology of each kind of fibers was not affected by the drug loading, their Ibuprofen release performance in an aqueous media was studied, expecting to take advantage of their different morphologies. All the samples were able to release all the loaded Ibuprofen but presented remarkable differences on the release rate. Solid PCL fibers presented a nonconstant and fast release rate, achieving the complete release after 7 hours. Hollow fibers also present a non-constant rate but required of almost one day to release all the Ibuprofen. Finally, hollow+porous fibers not only achieved a higher lbuprofen load but also showed a rather constant and slow release rate, keeping the Ibuprofen release for one day and a half. These different behaviors seem to be related to the roughness of the outer surfaces of the fibers, which were measured by AFM, determining that the hollow+porous fibers presented the higher roughness. According to their Ibuprofen release properties, the hollow+porous PCL microfibers obtained could be suitable candidates for the development of wound-dressing with a controlled local drug delivery capability.

\section{Conflicts of interest}

There are no conflicts to declare.

\section{Acknowledgements}

Financial assistance from MINECO, FEDER, UE (RTI2018-098749-B100, and RTI2018-097367-A-I00) and the Junta de Castile and Leon (VA275P18) are gratefully acknowledged. Moreover, postdoctoral contract of S. B. S. (Junta de Castile and Leon (VA275P18)) is acknowledged.

\section{References}

1 S. Lee, B. Kim, S. H. Kim, E. Kim and J. H. Jang, Adv. Funct. Mater., 2017, 27, 1-12.

2 D. Li and Y. Xia, Adv. Mater., 2004, 16, 1151-1170.

3 A. El-Fiqi, J. H. Kim and H. W. Kim, ACS Appl. Mater. Interfaces, 2015, 7, 1140-1152.

4 J. Xue, Y. Niu, M. Gong, R. Shi, D. Chen, L. Zhang and Y. Lvov, ACS Nano, 2015, 9, 1600-1612.

5 Y. Lu, J. Huang, G. Yu, R. Cardenas, S. Wei, E. K. Wujcik and Z. Guo, Wiley Interdiscip. Rev. Nanomedicine Nanobiotechnology, 2016, 8, 654-677.

6 R. Murugan and S. Ramakrishna, Tissue Eng., 2007, 13, 1845-1866.

7 Y. Xiang, J. Liang, L. Liu, F. Wang, L. Deng and W. Cui, ACS Appl. Mater. Interfaces, 2019, 11, 7836-7849.

Y. Ding, W. Li, F. Zhang, Z. Liu, N. Zanjanizadeh Ezazi, D. Liu 
and H. A. Santos, Adv. Funct. Mater., 2019, 29, 1-35. M. Norouzi, S. M. Boroujeni, N. Omidvarkordshouli and M. Soleimani, Adv. Healthc. Mater., 2015, 4, 1114-1133. R. Zhao, T. Ma, S. Li, Y. Tian and G. Zhu, ACS Appl. Mater. Interfaces, 2019, 11, 16662-16673.

11 S. Barroso-Solares, J. Pinto, G. Nanni, D. Fragouli and A. Athanassiou, RSC Adv., 2018, 8, 7641-7650.

12 S. Barroso-Solares, M. G. Zahedi, J. Pinto, G. Nanni, D. Fragouli and A. Athanassiou, RSC Adv., 2016, 6, 7110071107.

13 L. Cao, Y. Si, X. Yin, J. Yu and B. Ding, ACS Appl. Mater. Interfaces, 2019, 11, 35333-35342. B. Wang, H. Zheng, M. W. Chang, Z. Ahmad and J. S. Li, Colloids Surfaces B Biointerfaces, 2016, 145, 757-767. G. Duan and A. Greiner, Macromol. Mater. Eng., 2019, 304, 2-6. I. Ullah Khan, M. H. D. Othman, A. F. Ismail, T. Matsuura, H. Hashim, N. A. H. M. Nordin, M. A. Rahman, J. Jaafar and A. Jilani, J. Nat. Gas Sci. Eng., 2018, 52, 215-234.

A. L. Ahmad, T. A. Otitoju and B. S. Ooi, J. Ind. Eng. Chem., , DOI:10.1016/j.jiec.2018.10.005.

Z. Razzaz, A. Mohebbi and D. Rodrigue, Cell. Polym., 2018, 37, 169-188.

L. Zhao, C. Wu, Z. Liu, Q. Zhang and X. Lu, J. Memb. Sci., 2016, 505, 82-91.

X. M. Tan and D. Rodrigue, Polymers (Basel)., , DOI:10.3390/polym11081310.

S.-T. Lee, Polymeric foams: Innovations in processes, technologies, and products, CRC Press Taylor \& Francis Group, Boca Raton (USA), 2017.

B. Notario, J. Pinto and M. A. Rodriguez-Perez, Prog. Mater. Sci., 2016, 78-79, 93-139.

S. Costeux, J. Appl. Polym. Sci., DOI:10.1002/app.4129310.1002/APP.41293.

D. Miller, P. Chatchaisucha and V. Kumar, Polymer (Guildf)., 2009, 50, 5576-5584.

S. Orsi, E. Di Maio, S. Iannace and P. A. Netti, Nano Res., 2014, 7, 1018-1026.

M. C. Gutiérrez, Z. Y. García-Carvajal, M. Jobbágy, F. Rubio, L. Yuste, F. Rojo, M. L. Ferrer and F. Del Monte, Adv. Funct. Mater., 2007, 17, 3505-3513.

J. E. Tengood, D. Maskarinec, R. Ridenour and S. R. Little, J. Biomed. Mater. Res. - Part A, 2012, 100 A, 817-826.

J. Siepmann and F. Siepmann, Int. J. Pharm., 2008, 364, 328-343.

H. Zhang, J. K. Jackson and M. Chiao, Adv. Funct. Mater., 2017, 27, 1-31.

O. S. Fenton, K. N. Olafson, P. S. Pillai, M. J. Mitchell and R. Langer, Adv. Mater., 2018, 30, 1-29.

J. Zhao, C. Lu, X. He, X. Zhang, W. Zhang and X. Zhang, ACS Appl. Mater. Interfaces, 2015, 7, 2607-2615.

Y. Zhang, J. Ding, B. Qi, W. Tao, J. Wang, C. Zhao, H. Peng and J. Shi, Adv. Funct. Mater., 2019, 29, 1-14.

Q. Zhang, J. H. Oh, C. H. Park, J. H. Baek, H. M. Ryoo and K. M. Woo, ACS Appl. Mater. Interfaces, 2017, 9, 7950-7963. A. Szentivanyi, T. Chakradeo, H. Zernetsch and B.

Glasmacher, Adv. Drug Deliv. Rev., 2011, 63, 209-220.
P. Mostafalu, G. Kiaee, G. Giatsidis, A. Khalilpour, M. Nabavinia, M. R. Dokmeci, S. Sonkusale, D. P. Orgill, A. Tamayol and A. Khademhosseini, Adv. Funct. Mater., 2017, 27, 1-10.

M. Champeau, J. M. Thomassin, T. Tassaing and C. Jérôme, J. Control. Release, 2015, 209, 248-259.

A. P. Tiwari, T. I. Hwang, J. M. Oh, B. Maharjan, S. Chun, B. S. Kim, M. K. Joshi, C. H. Park and C. S. Kim, ACS Appl. Mater. Interfaces, 2018, 10, 20256-20270.

J. Pinto, J. A. Reglero-Ruiz, M. Dumon and M. A. RodriguezPerez, J. Supercrit. Fluids, 2014, 94, 198-205.

T. Hu, G. Dong, H. Li and V. Chen, J. Memb. Sci., 2014, 468, 107-117.

P. Huang, F. Wu, B. Shen, X. Ma, Y. Zhao, M. Wu, J. Wang, Z. Liu, H. Luo and W. Zheng, Chem. Eng. J., 2019, 370, 1322-1330.

J. Pinto, M. Dumon, M. Pedros, J. Reglero and M. A. Rodriguez-Perez, Chem. Eng. J., 2014, 243, 428-435. Y. He, F. Rezaei, S. Kapila and A. A. Rownaghi, ACS Appl. Mater. Interfaces, 2017, 9, 16288-16295.

A. De Rovere, B. P. Grady and R. L. Shambaugh, J. Appl. Polym. Sci., 2002, 83, 1759-1772.

Y. A. Hussain and C. S. Grant, J. Supercrit. Fluids, 2012, 71, 127-135.

S. L. Ma, Z. W. Lu, Y. T. Wu and Z. B. Zhang, J. Supercrit. Fluids, 2010, 54, 129-136.

R. Yoganathan, R. Mammucari and N. R. Foster, J. Phys. Conf. Ser., , DOI:10.1088/1742-6596/215/1/012087. 232, 81-90.

Y. Fu and W. J. Kao, Expert Opin. Drug Deliv., 2010, 7, 429444.

Q. Bénard, M. Fois and M. Grisel, Appl. Surf. Sci., 2007, 253, 4753-4758.

A. B. D. Cassie and S. Baxter, Trans. Faraday Soc., 1944, 40, 546-551. K. J. Kubiak, M. C. T. Wilson, T. G. Mathia and P. Carval, Wear, 2011, 271, 523-528.

A. Borras and A. R. González-Elipe, Langmuir, 2010, 26, 15875-15882.

V. Kumar and N. P. Suh, Polym. Eng. Sci., 1990, 30, 13231329.

F. F. . Martini-Vvedensky, J.J.E.; Suh, N.N.P.; Waldman, . M. Charoenchaitrakool, F. Dehghani, N. R. Foster and H. K. Chan, Ind. Eng. Chem. Res., 2000, 39, 4794-4802. Zhengwei Zhou; Jia Qian Jiang, J. Appl. Spectrosc., 2012, 79, 459-460. 